\title{
Desarrollo de morteros sostenibles para su aplicación en impresión 3D
}

\section{Development of sustainable mortars for application in 3D}

\section{printing}

Presentación: 15/10/2019

\author{
Doctorando:
}

\begin{abstract}
Adrián Isidro Yoris
Universidad Tecnológica Nacional - Universidad de Cantabria

ayoris@frsf.utn.edu.ar - yorisai@unican.es
\end{abstract}

Director/es:

\section{María Josefina Positieri - Elena Blanco Fernandez - Bárbara Belén Raggiotti}

\section{Resumen}

En el presente trabajo, correspondiente a la tesis doctoral en curso, se darán a conocer al gunos resultados de ensayos mecánicos y físicos de distintos tipos de morteros de geopolímeros y de cemento, cuya finalidad es ser utilizados como material aditivo en una impresora 3D, tipo contour crafting. Los morteros de geopolímeros empleados se componen de fly ash, como precursor, disolución alcalina, como activador, árido calizo, vidrio triturado y conchas marinas, como árido y aditivos. Los morteros de cemento, como su nombre lo indica, se componen de cemento, adiciones, aditivos y distintos áridos (arena caliza, vidrio triturado y conchas marinas). Los ensayos mecánicos corresponden a flexión y compresión en probetas prismáticas de 4 × 4 × 16 cm. Los morteros de geopolímero fueron curados en diferentes condiciones (a temperatura ambiente, en agua-de mar y de grifo- y en estufa a $40^{\circ} \mathrm{C}$ ) y se expusieron a diferentes ambientes (ambiente de laboratorio einmersos en agua); en tanto que los morteros de cemento se curaron a temperatura ambiente y en agua (de mar y de grifo). Se imprimieron elementos de diferentes tamaños, formas y complejidades para comprobar su efectividad como material aditivo, obteniéndose comportamientos satisfactorios. Los resultados mecánicos muestran que los morteros siguen adquiriendo resistencia hasta los 90 días, para cementos y 60 días para geopolímeros; estos últimos presentan menor resistencia comparados con los cementos, los cuales registraron valores entorno a los 70 MPa para los que contienen árido calizo y vidrio triturado, curados en agua.

Palabras claves: Mortero de cemento, Mortero de geopolímero, Impresión 3D, Sosteniblidad.

\begin{abstract}
In the present work, corresponding to the current doctoral thesis, some results of mechanical and physical tests of different types of geopolymer and cement mortars will be announced, whose purpose is to be used as additive material in a 3D printer, contour crafting type. The geopolymer mortars used are composed of fly ash, as a precursor, alkaline solution, as an activator, limestone aggregate, crushed glass and seashells, such as aggregate and additives. Cement mortars, as the name implies, consist of cement, additions, additives and various aggregates (limestone sand, crushed glass and seashells). The mechanical tests correspond to flexion and compression in prismatic specimens of $4 \times 4 \times 16 \mathrm{~cm}$. The geopolymer mortars were cured under different conditions (at room temperature, in water -sea and tap- and in a stove at $40^{\circ} \mathrm{C}$ ) and were exposed to different environments (laboratory environment and immersed in water); while cement mortars were cured at room temperature and in water (sea and tap). Elements of different sizes, shapes and complexities were printed to prove their effectiveness as an additive material, obtaining satisfactory behaviors. Mechanical results show that
\end{abstract}


mortars continue to gain resistance up to 90 days, for cement and 60 days for geopolymers; the latter have lower resistance compared to cements, which recorded values around $70 \mathrm{MPa}$ for those containing limestoneaggregate and crushed glass, cured in water.

Keywords: Cement mortar, Geopolymer mortar, 3D print, Sustainabiliy.

\section{Introducción}

La presente tesis doctoral se enmarca en un proyecto europeo denominado "Artificial Reef 3D Printing for Atlantic Area (3DPARE)", el cual, tiene como objetivo, promover la recuperación ambiental en obras marítimas o zonas costeras degradadas, por medio de la fabricación de arrecifes artificiales, mediante impresión 3D con morteros sostenibles.

Para la elección de los morteros sostenibles, se optó por morteros de geopolímeros y morteros de cemento con bajo contenido en Clinker. Los morteros de geopolímero se justifican considerando que hacen uso de desechos o subproductos industriales, que contienen aluminosilicatos para ser usados como precursor, los cuales, al ser combinados con una solución al calina, disuelven los óxidos de aluminio y de sílice, y geopolimerizan [1].

Por su parte, para los morteros de cemento, se escogió un cemento tipo III/B 32,5 N-SR, el cual es un cemento de al to horno, de resistencia media $32,5 \mathrm{MPa}$ a 28 días, con resistencia inicial normal [N] y resistente a sulfatos [SR] [2]. El mismo, tiene un contenido de escoria siderúrgica entre un $66 \%$ y $80 \%$, según noma, aunque lo habitual es que ronde el $66 \%$, lo que se traduce en un contenido de Clinker muy bajo, entorno al $31 \%$.

Los áridos utilizados en ambos tipos de morteros, fueron: arena caliza [0-3 mm], vidrio triturado [0-0,3 mm] y cochas marinas trituradas [0-1 mm]. El árido calizo proviene de la trituración de la roca caliza, y en España es un material abundante y de muy bajo costo. Por su parte, el vidrio triturado proviene de una empresa dedicada al reciclaje, en la cual, la fracción 0-0,3 mm representa un descarte, ya que al utilizar esta fracción para volver a obtener vidrio, presenta dificultades en el proceso de fundición [3]. Por último, las cochas marinas se obtienen como un residuo de la producción de pesca y cultivo de moluscos, fundamentalmente en la zona de Galicia, y para proceder a su uso, es necesario quitarle sus partes orgánicas mediante lavado con agua y luego proceder a su trituración y cribado [4], [5].

En esta investigación, el fly ash es utilizado como precursor en los geopolímeros y como adición en los morteros. A nivel mundial, el fly ash se reconoce como una de las fuentes más importantes de materia prima, representado por un total de 600 millones de toneladas por año. Este material se produce en las centrales eléctricas producto de la quema del carbón, y debido a su composición de aluminosilicatos, tamaño fino, gran cantidad de contenido vítreo y fundamental mente, su disponibilidad en todo el mundo, hace que sea uno de los materiales más empleados en la elaboración de geopolímeros [1].

Como activador, en los morteros de geopolímeros, se utilizó una disolución al calina. La determinación del uso de este activador, se llevó adelante mediante ensayos de prueba y error, mediante la elaboración de probetas prismáticas de $4 \times 4 \times 16 \mathrm{~cm}$ que se sometieron a flexión y compresión a las edades de 1,7 y 28 días. Además, en forma simultánea, se buscaron propiedades reológicas adecuadas para que los morteros obtenidos puedan ser extruidos correctamente en la impresora 3D [6], [7]. Una vez definido el activador, se elaboraron probetas prismáticas (que luego fueron sometidas a ensayos de compresión) con concentraciones de $\mathrm{NaOH}$ desde $8 \mathrm{M}$ a $16 \mathrm{M}$, definiéndose por resistencia e imprimibilidad del mortero, la concentración más adecuada [8].

Para conocer las propiedades de los morteros que se planteaba utilizar, se escogieron 6 dosificaciones, 3 correspondientes a morteros de geopolímeros y 3 a morteros de cemento, y se planteó una batería de ensayos en la cual se consideraran diferentes edades de ensayo y distintas condiciones de curado y de exposición. Se analizaron en forma alternativa, otras dosificaciones que no se expondrán aquí, haciendo uso de otros tipos de 
vidrios reciclados y deárido de residuos de la construcción y demolición (RCD), pero que por su baja resistencia no fueron tenidos en cuenta. En forma preliminar, además, se elaboraron más de 150 dosificaciones hasta llegar a las que se escogieron finalmente.

Los morteros de cemento a analizar difieren fundamentalmente en el tipo de árido utilizado: árido calizo, mezcla de árido calizo y conchas marinas, mezcla de árido calizo y vidrio triturado. Los morteros de geopolímeros, por su parte, se componen de los mismos áridos, pero difieren en las proporciones en el caso de la mezcla de árido calizo y vidrio triturado. Adicionalmente a las 6 dosificaciones a estudiar, se planteó una dosificación patrón, elaborada según norma, con el fin de caracterizar el cemento mecánicamente [9].

Todas las probetas se desmoldaron a las $24 \mathrm{~h}$ de haber sido mol deadas, a excepción de los morteros de cemento con conchas marinas, que requirieron un tiempo de $48 \mathrm{~h}$ debido a que el mismo, no había endurecido aún. Para Ilevar adelante los ensayos mecánicos se siguieron las recomendaciones de la norma UNE-EN 196-1 [9].

Teniendo en cuenta las variantes en los morteros, se plantearon las siguientes nomenclaturas para las dosificaciones:

CL: Cement Limestone (árido calizo)

CS: Cement Shells (con conchas marinas)

CG: Cement Glass (con vidrio triturado)

CP: probeta patrón de cemento

GL: Geopolymer limestone (árido calizo)

GS: Geopolymer Shells (con conchas marinas)

GG: Geopolymer Glass (con vidrio triturado)

Las edades de ensayo se fijaron en $1,3,7,28,63,91,182$ y 364 días de edad para todos los morteros y las condiciones de curado fueron: al aire e inmersas en agua (de grifo y de mar), para los morteros de cemento y al aire, al agua (de grifo y de mar) y a temperatura ( $40^{\circ} \mathrm{C}$ en estufa por $48 \mathrm{~h}$ ), para los morteros de geopolímeros. Por último, las condiciones de exposición para ambos morteros fueron: al aire y al agua (de grifo y de mar). El agua de mar fue extraída del mar Cantábrico, frente a las costas de la ciudad de Santander. Considerando las variables mencionadas anteriormente, se definieron las siguientes nomenclaturas:

CA: Cured Air (colocadas en ambiente de laboratorio desde el primer día edad)

CW: Cured Water (inmersas en agua de grifo desde el primer día de edad)

CSW: Cured Sea Water (inmersas en agua de mar desde el primer día de edad)

T40: Temperature $40^{\circ} \mathrm{C}$ (curado en estufa a $40^{\circ} \mathrm{C}$ por $48 \mathrm{~h}$, luego de haber sido desmoldadas)

IW7: I mmersion Water 7 dais (se sumergen en agua de grifo luego de haber estado hasta los 7 días de edad en ambiente de laboratorio)

pI W: previous I mmersion Water (se sumergen en agua de grifo por $24 \mathrm{~h}$, el día previo al ensayo, luego dehaber estado en ambiente de laboratorio)

Debido a que no todas las dosificaciones de morteros se someten a todas las variantes de curado y exposición, se generan las siguientes combinaciones:

\begin{tabular}{|c|c|c|c|c|c|c|}
\hline CL-CA & CL-CW & CL-CSW & CS-CA & CS-CW & CG-CA & CG-CW \\
\hline GL-CA & GL-CW & GL-CSW & GL-T40 & GL-IW7 & GL-pIW & \\
\hline GS-CA & GS-T40 & GS-IW7 & GSpIW & GG-CA & GG-T40 & GG-IW7 \\
\hline
\end{tabular}

Las distintas dosificaciones se componen de los siguientes materiales:

CL: cemento, árido calizo, adiciones, aditivo y agua. [a/c=0.53].

CS: cemento, árido calizo, conchas marinas, adiciones, aditivo y agua. [a/c=0.63].

CG: cemento, árido calizo, vidrio triturado, adiciones, aditivo y agua. [a/c=0.57].

CP: cemento (450g), árido calizo (1350 g) y agua (225g).

GL: fly ash, disolución alcalina, agua, árido calizo y aditivos.

GS: fly ash, disolución alcalina, agua, árido calizo, conchas marinas y aditivos. 
GG: fly ash, disolución alcalina, agua, árido calizo vidrio triturado y aditivos.

\section{Resultados}

Las Figuras 1 a 5 presentan los resultados de los ensayos a flexión y compresión de los morteros evaluados, sometidos a diferentes curados y exposiciones. No se muestran los resultados de todas las edades, ya que la elaboración de las probetas se realizó en forma diferida en el tiempo y aún se encuentran en proceso de evaluación. En forma general, se observa que los morteros de cemento CA presentan menor resistencia mecánica que los morteros curados en agua; pero no ocurre lo mismo con las probetas GL-CA y GL-T40, este comportamiento se da en forma inversa, las probetas CA y T 40 presentan mejores resistencias mecánicas que las probetas curadas en agua, sin embargo, en los morteros GG y GS no existe una marcada diferencia entre las resistencias mecánicas según las condiciones de curado y exposición.

En la Figura 1 se observa que, a los 91 días de edad, las probetas CW adquieren 70 MPa de resistencia a compresión, en tanto que, para CA, la resistencia es $30 \%$ menos; haciéndose notable esta diferencia a partir de los 7 días de edad, en compresión y a partir del primer día, en flexión. Luego de los 28 días, las probetas CW superan en compresión a las probetas patrón. Además, la resistencia de las probetas CSW, supera levemente a las CW.

En la Figura 2, se observa que, a partir de los 7 días de edad, las probetas CS-CW aumentan su resistencia mecánica en forma considerable, llegando a superar los $50 \mathrm{MPa}$ a los 91 días, en tanto que, para la misma edad, las probetas CA tienen un $40 \%$ menos de resistencia. Lo propio sucede con los morteros CG, donde las probetas CW Ilegan a valores próximos a los de las probetas CL-CW.
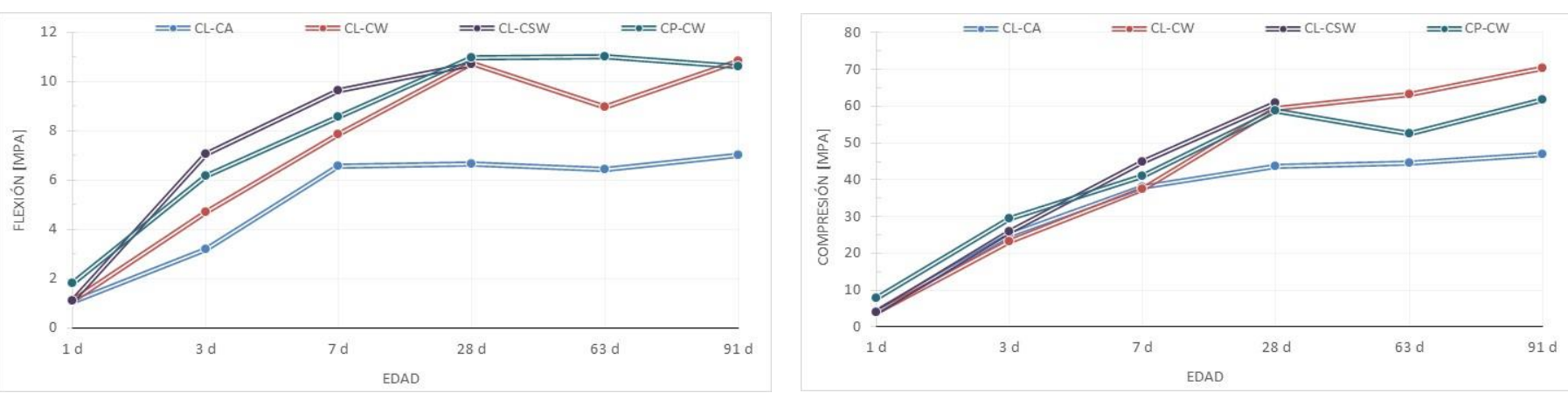

Figura 1. Ensayos de flexión y compresión de los morteros CL y CP
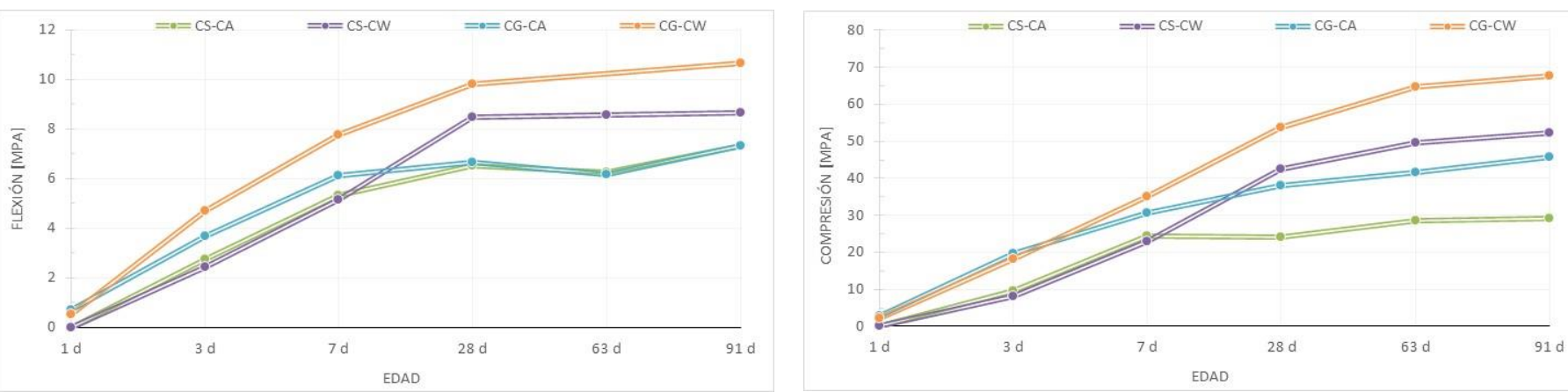

Figura 2. Ensayos de flexión y compresión de los morteros CS y CG

En las Figuras 3 a 5, se presentan los resultados de los ensayos de los morteros de geopolímeros, pudiéndose observar en todos ellos, que las probetas T40 elevan su resistencia en las primeras edades, pero a partir de los 28 días, tienden a asemejarse a las CA. En el caso particular de los morteros GL, las probetas CA alcanzan resistencias a compresión cercana a los $40 \mathrm{MPa}$, en tanto que las T40 se mantienen en los $32 \mathrm{MPa}$.

Para los morteros GL, las probetas pIW presentan una disminución considerable en las resistencias mecánicas [Figura 3]; para la edad de 63 días en compresión, esta disminución representa 35 \% aproximadamente, lo que 
demuestra una importante afectación por exposición a condiciones de mojado, aunque sea por períodos cortos. Sin embargo, este comportamiento no se repite en los morteros GG y GS [Figuras 4 y 5], donde las probetas pI W, tienen resistencias a compresión levemente superior a CA.

Como sucede en los morteros de cemento, en los morteros GL [Figura 3], las probetas CSW presentan resistencias mecánicas levemente superior a CW, pero ambas, son muy inferiores a CA. Para la edad de 28 días, las probetas CA adquieren resistencia a compresión superior a los $30 \mathrm{MPa}$, en tanto que las CSW y CW se encuentran próximas a los $10 \mathrm{MPa}$.

Para todos los casos [Figuras 3, 4 y 5], en las probetas IW7 se pueden observar en forma preliminar, valores de resistencia mecánica levemente inferiores a las pl W, notándose diferencias más acentuadas para los morteros $\mathrm{GL}$, donde a los 28 días de edad, disminuye un $25 \%$.
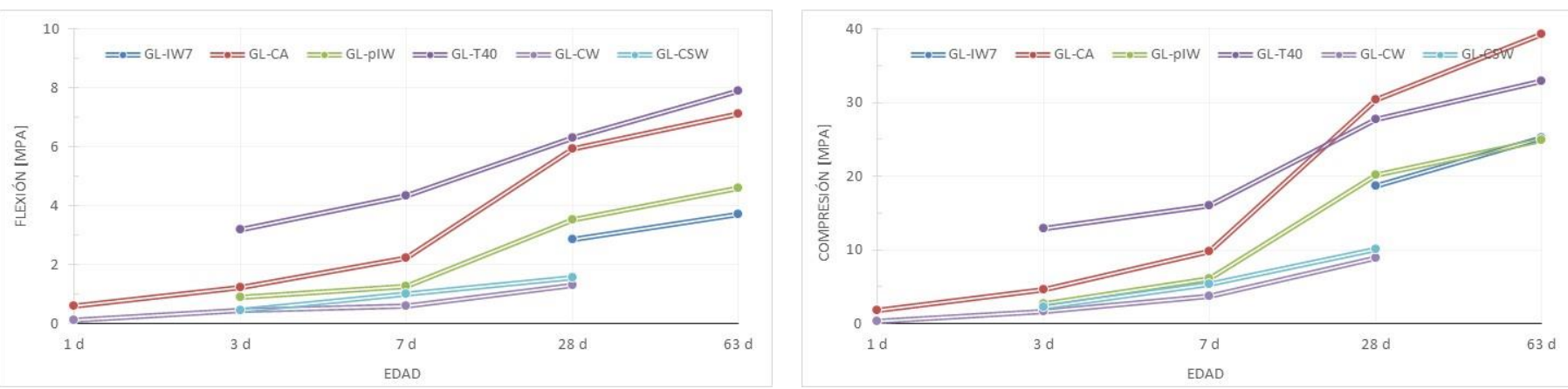

Figura 3. Ensayos de flexión y compresión de los morteros GL
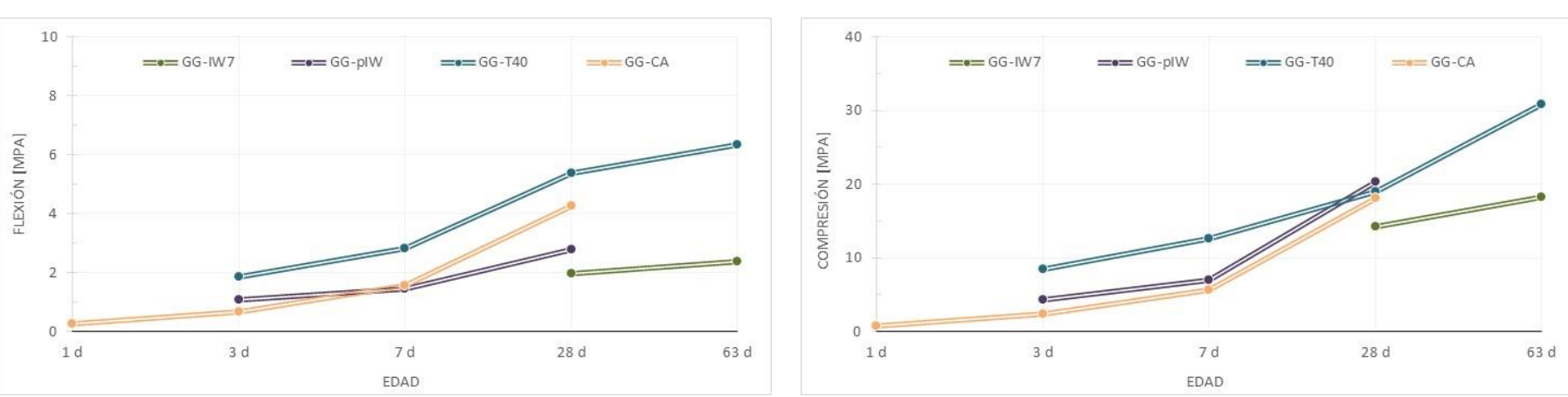

Figura 4. Ensayos de flexión y compresión de los morteros GG
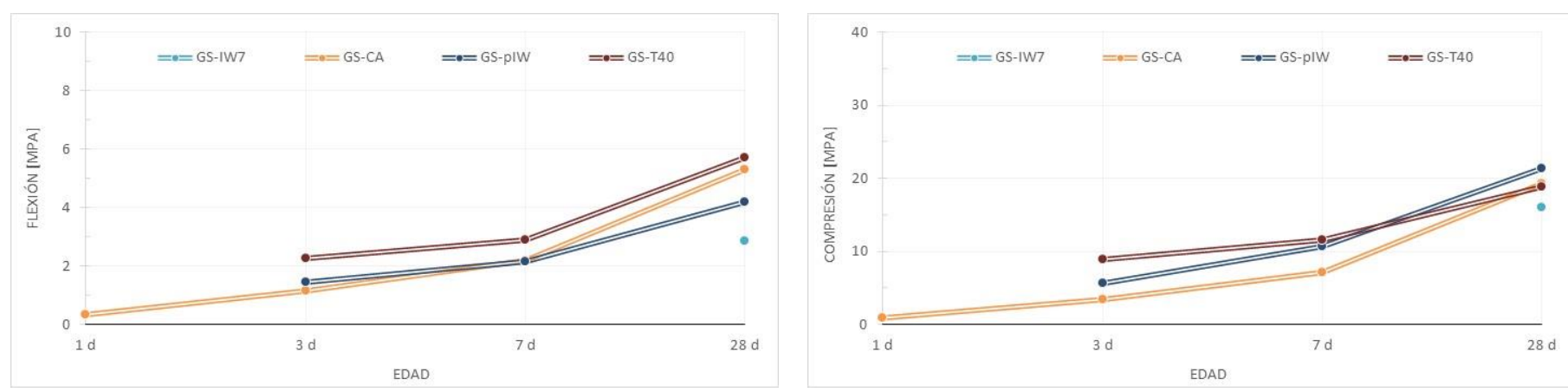

Figura 5. Ensayos de flexión y compresión de los morteros GS

En la Figura 6, se presentan imágenes de dos elementos, resultado de pruebas preliminares de una parte de los modelos de arrecifes artificiales para el proyecto 3DPARE. En ellas, se puede observar que la definición y continuidad de las capas de morteros es muy buena, lo que habla por sí solo de que se logró dar con un mortero de consistencia adecuada para imprimir este tipo de piezas. 

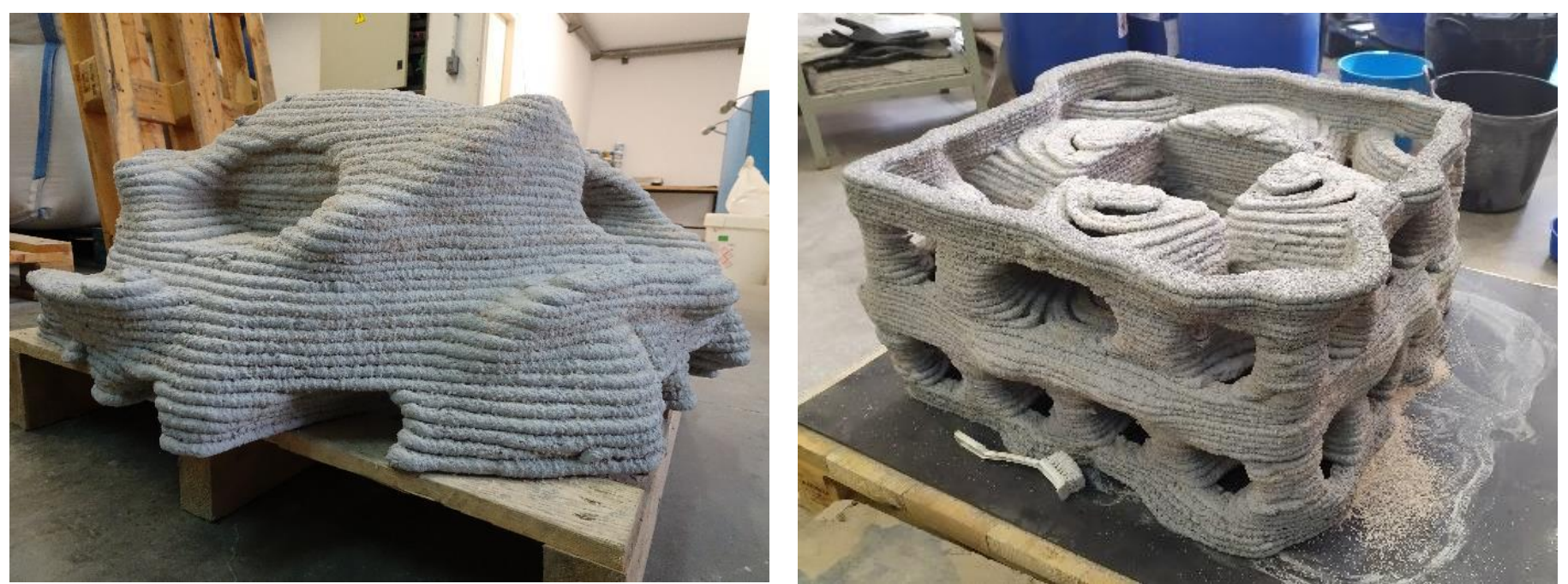

Figura 6. Parte de los model os de arrecifes artificiales impresos con el mortero CL

El siguiente paso en el proyecto, próximo a comenzar, es la elaboración de 32 piezas correspondientes a los arrecifes artificiales, para los cuales se emplearán dos tipos de morteros (a definir). Se elaborarán dos formas (cilíndrica y cúbica) con dos tipos de terminación superficial (pequeñas y grandes rugosidades). Esto representará la factibilidad del uso de los morteros desarrollados y de las técnicas a emplear, para la elaboración de piezas de formas irregulares y complejas.

Respecto a la investigación, quedan por realizar varios ensayos y pruebas aún, entre los más importantes están los siguientes:

- Continuar con la caracterización física y química de los materiales empleados en los morteros.

- Continuar con la caracterización mecánica de los morteros.

- Conocer la durabilidad de los morteros mediante penetración de agua y permeabilidad al oxígeno.

- Elaborar probetas impresas para caracterizarlas física y mecánicamente (flexión, compresión, tracción).

- Relacionar la reología de los morteros en estado fresco con la imprimibilidad de los mismos.

- Conocer el impacto ambiental de la producción de los morteros, mediante un análisis de ciclo de vida.

- Y finalmente, reproducir estos morteros en Argentina haciendo uso de materiales locales y buscando optimizar las dosificaciones para aumentar la sustentabilidad y disminuir el impacto ambiental de su producción.

\section{Referencias}

[1] T. Xie and T. Ozbakkaloglu, "Behavior of low-calcium fly and bottom ash-based geopolymer concrete cured at ambient temperature,” Ceram. Int., vol. 41, no. 4, pp. 5945-5958, May 2015.

[2] UNE-EN 197-1. Cemento. Parte 1: Composición, especificaciones y criterios de conformidad de los cementos comunes. AENOR - Asociación Española de Normalización y Certificación, 2011.

[3] M. Vafaei and A. Allahverdi, "High strength geopolymer binder based on waste-glass powder," Adv. Powder Technol., vol. 28, no. 1, pp. 215-222, Jan. 2017.

[4] R. Rodríguez-Álvaro, "Morteros para revestimiento con árido procedente de concha de mejillón," Universidade da Coruña, 2014.

[5] C. Varhen, S. Carrillo, and G. Ruiz, "Experimental investigation of Peruvian scallop used as fine aggregate in concrete,” Constr. Build. M ater., vol. 136, pp. 533-540, Apr. 2017.

[6] B. Panda, S. C. Paul, N. A. N. Mohamed, Y. W. D. Tay, and M. J. Tan, "Measurement of tensile bond strength of 3D printed geopolymer mortar,” M easurement, vol. 113, pp. 108-116, Jan. 2018.

[7] R. A. Buswell, W. R. Leal de Silva, S. Z. Jones, and J. Dirrenberger, "3D printing using concrete extrusion: A roadmap for research,” Cem. Concr. Res., vol. 112, pp. 37-49, Oct. 2018. 
[8] G. Görhan and G. Kürklü, "The influence of the $\mathrm{NaOH}$ solution on the properties of the fly ash-based geopolymer mortar cured at different temperatures," Compos. Part B Eng., vol. 58, pp. 371-377, Mar. 2014.

[9] UNE-EN 196-1. M étodos de ensayo de cementos Parte 1: Determinación de resistencias. AENOR - Asociación Española de Normalización y Certificación, 2018. 